\title{
The lower the volume of a cleansing product the higher its osmolarity and thus the risk of determining electrolyte imbalances in predisposed patients
}

다(1)우우

\author{
Authors \\ Mario Schettino, Alessia Dalila Guarino, Gianpiero Manes \\ Institution \\ Gastroenterology and Digestive Endoscopy Unit, ASST \\ Rhodense, Garbagnate Milanese and Rho Hospitals, Milan, \\ Italy \\ Bibliography \\ Endosc Int Open 2021; 09: E1205-E1206 \\ DOI 10.1055/a-1487-5876 \\ ISSN 2364-3722 \\ (c) 2021. The Author(s). \\ This is an open access article published by Thieme under the terms of the Creative \\ Commons Attribution-NonDerivative-NonCommercial License, permitting copying \\ and reproduction so long as the original work is given appropriate credit. Contents
}

\author{
may not be used for commercial purposes, or adapted, remixed, transformed or \\ built upon. (https://creativecommons.org/licenses/by-nc-nd/4.0/) \\ Georg Thieme Verlag KG, Rüdigerstraße 14, \\ 70469 Stuttgart, Germany \\ Correspondence \\ Gianpiero Manes, MD, Gastroenterology and Digestive \\ Endoscopy Unit, ASST Rhodense, Garbagnate Milanese and \\ Rho Hospitals, Viale Forlanini 95, Garbagnate Milanese, Milan, \\ Italy \\ Fax: +3902994302905 \\ gimanes@tin.it
}

Because colorectal cancer (CRC) represents a significant cause of mortality worldwide and incidence is rising in younger people, particular importance is given to effective screening programs, including noninvasive and invasive modalities [1]. In particular, colonoscopy has a central role in detecting earlystage CRC and identifying and resecting precancerous lesions. A quality examination requires adequate bowel preparation, considering that poor colon cleansing negatively influences quality indicators for colonoscopy, such as adenoma detection rate and cecal intubation. The theme of ideal bowel preparation is still controversial, because it should meet criteria of tolerability and efficacy, but safety also is a concern, particularly in fragile patients [2].

The efficacy of low-volume and very-low-volume polyethylene glycol (PEG)-based bowel preparations is well-established. Several studies and meta-analyses comparing PEG-based preparations of different volumes and different characteristics have reported similar efficacy for overall colon cleansing. [3, 4] The recent trend toward the development of the lowest-volume bowel preparations, obtained with the adjunct of osmotically active substances such as ascorbic acid, has drawn attention to the safety of these solutions. Because of their high osmolarity, low-volume bowel preparations are likely more prone to influence fluid and electrolyte balance.

Because major concerns have been raised about the occurrence of electrolyte imbalance and dehydration following the ingestion of bowel preparation, large-volume, iso-osmotical, PEG-based bowel preparations have been developed and have become the standard of care in the last decades. In general, electrolyte alterations and dehydration may be caused by different mechanisms induced by bowel preparations, such as diarrhea, but colonoscopy itself activated the renin-angiotensin-aldosterone system. Hypokalemia may present with a wide spectrum of clinical characteristics, ranging from absence of symptoms to constitutional symptoms such as fatigue, muscular weakness, arrhythmias, and cardiac arrest. The clinical implications are related not only to the severity of hypokalemia and the rapidity of onset, but also to the characteristics of patients, because the major incidence of hypokalemia in hospitalized and elderly patients or patients on thiazide therapy is well known $[5,6]$. The use of sodium phosphate as bowel preparation, for example, has been associated with disruption in electrolytes homeostasis, including hypokalemia [7,8]. High-volume PEG solutions are generally considered safe, even in patients who are at risk for serum electrolyte imbalance, and accordingly, European Society of Gastrointestinal Endoscopy guidelines suggest that in patients at risk for hydroelectrolyte disturbances, the choice of laxative should be individualized, with large-volume PEG products still playing an important role, while attention must be paid if low-volume hyperosmotic formulations are used [9].

In this issue of Endoscopy International Open, Reumkens et al. have published a prospective cohort study addressing the problem of the risk of developing hypokalemia following the ingestion of a low-volume PEG-ascorbic acid solution as bowel preparation for colonoscopy. 
Although no serious adverse event occurred, about $5 \%$ of normokalemic individuals developed hypokalemia after bowel preparation and $1 \%$ of the initial population presented with a combination of both a "high cardiac risk" profile and hypokalemia. Female sex, thiazide use, and CRC diagnosis were found to be predictors of hypokalemia development. The authors conclude that additional screening for electrolyte imbalances may be needed in "high cardiac risk" patients and those on thiazide diuretics who are more prone to develop post-cleansing hypokalemia.

In a previous study, performed in patients considered at risk for hypokalemia, the authors of the current paper found that $23.6 \%$ of patients developed hypokalemia after bowel preparation with low-volume PEG plus ascorbic acid solution [6]. Similar results were observed by $\mathrm{Ho}$ et al in their retrospective chart review, which found a rate of $20.5 \%$ of hypokalemia after administration of high-volume PEG solution in hospitalized patients aged $\geq 65$ years [10].

The data, thus, are still sparse and equivocal. In a randomized controlled trial (RCT) comparing 4-L PEG and 2-L PEG-ascorbic acid solutions, the levels of serum potassium decreased after intake of 4-L PEG compared to 2-L PEG [11]. Moreover, from the literature review, no univocal pattern emerges of electrolyte changes after bowel preparations; however, almost all of the studies agree that these changes are transient and of uncertain clinical significance [11-14].

Undoubtedly, the adjunct of osmotically active substances to bowel preparation solutions increases the risk of dehydration and impairs electrolyte balance. A recently published metanalysis that included 3 RCTs comparing a 1-L PEG-based preparation for colonoscopy (NER1006) to trisulfate, sodium picosulfate plus magnesium citrate, and 2-L PEG preparations, showed a higher incidence of dehydration in NER1006 [4].

At present, the market trend is extremely oriented toward the use of very-low $(1 \mathrm{~L})$, hyperosmotic, PEG-based preparations because they are very effective and well accepted by patients; their use will likely increase in coming years. This fact prompts the need for further real-life studies to properly investigate the safety of low-volume bowel preparations, especially in at-risk populations; in particular, the real impact of the transient dehydration and electrolyte imbalances induced by bowel preparation should be explored. At the same time, availability of different products with different characteristics should push endoscopists to make an effort to individualize colon preparation to minimize the risk of adverse events, as suggested by the current guidelines [9].

\section{Competing interests}

The authors declare that they have no conflict of interest.

\section{References}

[1] Ladabaum U, Dominitz JA, Kahi C et al. Strategies for colorectal cancer screening. Gastroenterology 2020; 158: 418-432

[2] Jang JY, Chun HJ. Bowel preparations as quality indicators for colonoscopy. World J Gastroenterol 2014; 20: 2746-2750

[3] Choe YG et al. Randomized trial of 2-L polyethylene glycol + ascorbic acid versus 4-L polyethylene glycol as bowel cleansing for colonoscopy in an optimal setting. J Gastroenterol Hepatol 2014; 29: 12231228

[4] Maida M, Macaluso FS, Sferrazza S et al. Effectiveness and safety of NER1006 versus standard bowel preparations: A meta-analysis of randomized phase-3 clinical trials. Dig Liver Dis 2020; 52: 833-839

[5] Marín Gabriel JC, Rodríguez Muñoz S, de la Cruz Bértolo J et al. Electrolytic disturbances and colonoscopy: bowel lavage solutions, age and procedure. Rev Esp Enferm Dig 2003; 95: 863-875

[6] Reumkens A, Masclee AA, Winkens B et al. Prevalence of hypokalemia before and after bowel preparation for colonoscopy in high-risk patients. Gastrointest Endosc 2017; 86: 673-679

[7] Brunelli SM. Association between oral sodium phosphate bowel preparations and kidney injury: a systematic review and meta-analysis. J Kidney Dis 2009; 53: 448-456

[8] Hookey LC, Depew WT, Vanner S. The safety profile of oral sodium phosphate for colonic cleansing before colonoscopy in adults. Gastrointest Endosc 2002; 56: 895-902

[9] Hassan C, East ], Radaelli F et al. Bowel preparation for colonoscopy: European Society of Gastrointestinal Endoscopy (ESGE) Guideline Update 2019. Endoscopy 2019; 51: 775-794

[10] Ho JM, Juurlink DN, Cavalcanti RB. Hypokalemia following polyethylene glycol-based bowel preparation for colonoscopy in older hospitalized patients with significant comorbidities. Ann Pharmacother 2010; 44: 466-470

[11] Lee KJ, Park HJ, Kim HS et al. Electrolyte changes after bowel preparation for colonoscopy: A randomized controlled multicenter trial. World J Gastroenterol 2015; 21: 3041-3048

[12] Bisschops R, Manning J, Clayton LB et al. Colon cleansing efficacy and safety with $1 \mathrm{~L}$ NER 1006 versus $2 \mathrm{~L}$ polyethylene glycol + ascorbate: a randomized phase 3 trial. Endoscopy 2019; 51: 60-72

[13] Rex DK, McGowan J, Cleveland MV et al. A randomized, controlled trial of oral sulfate solution plus polyethylene glycol as a bowel preparation for colonoscopy. Gastrointest Endosc 2014; 80: 482-491

[14] Mathus-Vliegen EM, van der Vliet K. Safety, patient's tolerance, and efficacy of a 2-liter vitamin C-enriched macrogol bowel preparation: a randomized, endoscopist-blinded prospective comparison with a 4-liter macrogol solution. Dis Colon Rectum 2013; 56: 1002-1012 